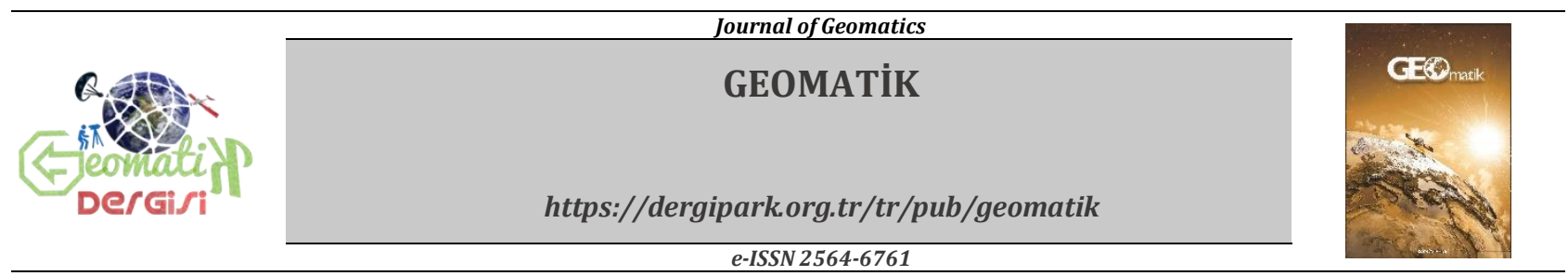

\title{
Farklı yazılımlar kullanılarak gerçek zamanlı hassas nokta konum belirleme (RT-PPP) yönteminin performansının incelenmesi
}

\author{
Salih Alçay ${ }^{1 *}$, Ömer Faruk Atiz ${ }^{1}$ \\ ${ }^{1}$ Necmettin Erbakan Üniversitesi, Mühendislik ve Mimarlık Fakültesi, Harita Mühendisliği Bölümü, Konya, Türkiye
}

\author{
Anahtar Kelimeler \\ $\mathrm{BKG} / \mathrm{BNC}$ \\ IGS/RTS \\ PPP-WIZARD \\ RT-PPP \\ RTKLIB/RTKNAVI
}

\begin{abstract}
ÖZ
Göreli konum belirleme yöntemine alternatif olarak ortaya çıkan Hassas Nokta Konum Belirleme (Precise Point Positioning-PPP) yöntemi son yıllarda yaygın olarak pek çok uygulamada kullanılmaktadır. PPP yöntemi mutlak konum belirleme yönteminin özel bir durumu olup, bu yöntemle tek bir GNSS alıcısı ile toplanan verilerden yararlanarak yüksek doğrulukla statik veya kinematik olarak konum belirlenebilmektedir. Diğer yandan gerçek zamanlı (real-time) hassas yörünge ve saat bilgilerinin farklı kuruluş ve organizasyonlar tarafından (International GNSS Service-IGS, Federal Agency for Cartography and Geodesy-BKG, European Space Agency-ESA, European Reference Frame-EUREF, German Research Centre for Geosciences-GFZ vb.) kullanıma sunulmaları sayesinde PPP yöntemi gerçek zamanlı konum bilgisi gerektiren uygulamalarda kullanılmaya başlanmıştır. Gerçek-zamanlı-PPP (RT-PPP) olarak adlandırılan bu yönteme olan ilginin oldukça yüksek olması yöntemin gelișimine hız katmıştır. Bu bağlamda birçok yazılım geliştirilmiștir. $\mathrm{Bu}$ çalıșmada, söz konusu bu yazılımlardan BKG/BNC (BKG Ntrip Client), RTKLIB/RTKNAVI (Real Time Kinematic Library) ve PPP-WIZARD (PPP With Integer and Zero-difference Ambiguity Resolution Demonstrator) kullanılarak, RT-PPP yönteminin konum belirleme performansı incelenmiştir. Bu amaçla IGS/RTS istasyonlarından İstanbul ilinde yer alan ISTA istasyonun yaklaşık 25 saatlik gözlem süresine ait gerçek zamanlı koordinat değerleri 1 saniye epok aralığında elde edilmiştir. Elde edilen sonuçlar noktanın referans kabul edilen koordinat değerleri ile karşılaştırılarak doğruluk ve hassasiyet bakımından incelenmiştir. Elde edilen sonuçlara göre, yakınsama süresinin zaman zaman 1 saatin üzerine çıktığı, bu sürenin sonrasında ise BKG/BNC ve RTKLIB/RTKNAVI ile yatay bileșende genellikle $\pm 10 \mathrm{~cm}$, düşey bileșende ise $\pm 20 \mathrm{~cm}$ civarında bir doğruluğun elde edilebildiği görülmüştür. Ancak PPP-WIZARD yazılımı kullanılarak elde edilen koordinatların doğruluğu çok sayıda oluşan bağlantı kesikliği ve sonrasındaki yakınsama süreci nedeniyle \pm 30 cm'nin üzerindedir.
\end{abstract}

\section{Investigation of the performance of Real-Time Precise Point Positioning (RT-PPP) method using different software packages}

Keywords
BKG/BNC
IGS/RTS
PPP-WIZARD
RT-PPP
RTKLIB/RTKNAVI

\begin{abstract}
The Precise Point Positioning technique (PPP), which emerged as an alternative to the conventional relative positioning method, has been widely used in many applications in recent years. The PPP method is a special case of absolute positioning method, in this method the static and kinematic position can be determined with high accuracy by using single GNSS receiver. On the other hand, thanks to the real-time precise orbit and clock products made available by different establishments and organizations (International GNSS Service-IGS, Federal Agency for Cartography and Geodesy-BKG, European Space Agency-ESA, European Reference FrameEUREF, German Research Centre for Geosciences-GFZ etc.), PPP method has started to be used in applications that require real-time position information. The high interest in this method, which is called real-time-PPP (RT-PPP), has accelerated the development of the method. Many software have been developed in this context. In this study, the positioning performance of RTPPP method was investigated using BKG/BNC (BKG Ntrip Client), RTKLIB/RTKNAVI (Real Time Kinematic Library) and PPP-WIZARD (PPP With Integer and Zero-difference Ambiguity Resolution Demonstrator) software. For this purpose, real time coordinate values of ISTA IGS station, located in Istanbul, belongs to the observation period of about 25 hours were obtained in 1 second epoch interval. The obtained results were compared with the reference coordinates of the station, and examined in terms of accuracy and precision. According to the results, it has been observed that the convergence time sometimes exceeds 1 hour, and after this time, an
\end{abstract}


accuracy of approximately $\pm 10 \mathrm{~cm}$ in horizontal component and $\pm 20 \mathrm{~cm}$ in vertical component could be obtained with BKG/BNC and RTKLIB/RTKNAVI. However the accuracy of the coordinates obtained using the PPP-WIZARD software is over $\pm 30 \mathrm{~cm}$ due to the large number of disconnections and subsequent convergence.

\section{GíRiş}

Bağll konum belirleme yöntemleri günümüzde jeodezik ölçmeler, tektonik hareketlerin belirlenmesi, baraj-köprü gibi mühendislik yapılarının izlenmesi, deformasyon ölçmeleri gibi yüksek doğruluk gerektiren birçok mühendislik uygulamasında yaygın olarak kullanılmaktadır. 1990'lı yıllarda geliştirilen Klasik-RTK (Real Time Kinematic) yöntemi gerçek zamanlı uygulamalarda da kullanılmaya başlanmıştır. İzleyen yıllarda CORS (Continuously Operating Reference Station) ağlarının gelişmesiyle birlikte Ağ-RTK (Network RTK) yöntemi, pek çok ülkede yaygın olarak kullanılır hale gelmiştir (Zhang ve ark. 2006; Martin ve McGovern 2012; Tsuji ve ark., 2017; Kahveci, 2017). Klasik-RTK yönteminde referans bir alıcı ile gezici alıcılar kullanılarak, oldukça kısa faz başlangıç belirsizliğinin çözüm süresi (initialization) sonrasında $\mathrm{cm}$ mertebesinde doğrulukla konum belirlenebilmektedir. Yöntemin doğruluğu uydu yörünge hatası, iyonosferik-troposferik etkilere bağlı olarak değişmektedir. Dolayısıyla klasikRTK'nın sağladığı konum doğruluğu referans ve alıcı arasındaki mesafeye bağlıdır (Rizos ve Han, 2003). Düzeltme bilgisi radyo bağlantısı aracilığıyla gerçekleştiğinde baz mesafesi yaklaşık $10 \mathrm{~km}$ ile sınırlı olup, Internet aracılığıyla sağlandığında ise daha uzak mesafelerde de yöntem kullanılabilmektedir. Klasik-RTK' da kullanıcının referans istasyon kurma zorunluluğunu ortadan kaldırmak için Ağ-RTK tekniği ortaya çıkmıştır ve birçok ülke kendi sistemini oluşturmuştur. Bu yöntemde ağdan hesaplanan düzeltmeler alıcıya Internet (GSM) bağlantısı yardımıyla iletilmektedir. Böylece gerçek zamanlı 3 boyutlu konum bilgisi $\mathrm{cm}$ seviyesindeki doğrulukla kullanıcıya sunulmaktadır. Ancak bu yöntemin öne çlkan en önemli dezavantajları, gerekli ağ altyapısının ve kullanılan donanımların gerek kurulum, gerekse de işletim maliyetlerinin yüksek olması, referans istasyonlardaki hataların doğrudan alıcının konumunu etkilemesi ve yöntemin Internet/GSM bağlantısı olmayan veya kısıtlı olan yerlerde çalışma yapılmasındaki kısıtlardır. Klasik-RTK ve Ăg-RTK yöntemleri ile ilgili daha detaylı bilgiler, Kahveci (2017)'de yer almaktadır.

Klasik-RTK ve Ağ-RTK tekniklerinde referans istasyon kullanma zorunluluğu olduğundan, son yıllarda mutlak konum belirleme yöntemlerinden PPP yöntemine olan ilgi artmıştır. Bu yöntemde tek bir GNSS alıcısı kullanılarak konum belirlenebildiğinden oldukça ekonomik bir yöntemdir. PPP yönteminin performansı uydu yörünge ve saat düzeltmelerinin kalitesine bağlıdır (Zhou ve ark., 2019). Ölçme sonrası veri işleme (Post-process) PPP yönteminin performansı üzerine birçok çalışma yapılmıştır (Soycan, 2012; Choy ve ark., 2013; Yiğit ve ark., 2014; Alkan ve ark., 2015; Alçay, 2016; Alcay ve ark., 2019; Ogutcu, 2020). Elde edilen sonuçlar statik ve kinematik PPP uygulamaları ile $\mathrm{cm}$-dm seviyesinde bir doğruluğun elde edilebileceğini göstermiştir. IGS-RT servisinin yörünge ve saat ürünlerini anlık olarak sunması, PPP yönteminin gerçek zamanlı olarak kullanılmasına olanak tanımıştır (Ahmed ve ark., 2016; Krzan ve Przestrzelski, 2016; Alçay, 2019). RT-PPP yönteminde tamsayı faz başlangıç belirsizliği çözümü (ambiguity-fixed) gerçekleştiğinde KlasikAğ RTK yöntemlerine alternatif bir yöntem olabileceği beklenmektedir (Grinter ve ark., 2020). $\mathrm{Bu}$ çalışmada RT-PPP yazılımlarından BKG/BNC, PPP-WIZARD ve RTKLIB/RTKNAVI kullanılarak yöntemin sağladığı konum doğruluğu detaylı olarak analiz edilmiştir.

\section{GERÇEK ZAMANLI (RT) PPP}

RT-PPP üzerine yoğunlaşan çalıșmalar neticesinde çok sayıda yazılım paketi üretilmiştir (Alçay, 2019). Bunlardan bazlları ücretli olup bazıları da ücretsiz olarak kullanıcının hizmetine sunulmuştur. Bu çalışmada kullanılan BKG / BNC, PPP-WIZARD, RTKLIB / RTKNAVI yazılımlarına ait detaylar aşağıda verilmiştir.

\subsection{BNC Yazılımı}

BNC yazılımı Federal Agency for Cartography and Geodesy (BKG) tarafindan geliştirilmiştir. Yazılım RT-PPP uygulamasını kod+faz gözlemlerini, yayın efemerisi ile uydu yörünge ve saati için düzeltme verilerini kullanarak gerçekleștirmektedir. Veri işleme aşamasında düzeltmeler anlık akış şeklinde yayın efemerisine uygulanmaktadır. Yazılım tüm aktif küresel uydu sistemlerini desteklemektedir. Yazılımın sunduğu hizmete ait detaylar URL-1'de verilmiştir.

\subsection{RTKLIB/RTKNAVI Yazılımı}

RTKLIB Tokyo Üniversitesi Deniz Bilimi ve Teknolojisi bölümü tarafından geliştirilen açık kaynak kodlu bir yazılım paketidir. Ölçme sonrası veri işleme (Post-process) ve gerçek zamanlı olmak üzere birçok GNSS uygulamasını (statik, kinematik, DGNSS, PPP vb.) desteklemektedir. Yazılım GPS, GLONASS, GALILEO, QZSS, BEIDOU ve SBAS sistemlerine ait verileri kullanabilmektedir. Ayrıca yazılımda standart GNSS veri protokolleri (RINEX, NMEA, RTCM) desteklenmektedir. Yazılım paketinin birçok modülü olup RT-PPP hizmeti veren modülü RTKNAVI' dir. Yazılıma ait detaylar Takasu 2013'de verilmiştir. 


\subsection{PPP-WIZARD}

PPP-WIZARD, Ulusal Uzay Araștırmaları Merkezi (CNES) tarafından geliştirilmiştir. Yazılım kod, faz, doppler gözlemlerini kullanabilmektedir. Diğer iki yazılımdan farklı olarak tam sayı belirsizlik çözümünü (AR) gerçekleştirebilmektedir. Uydu sistemlerinden GPS ve GLONASS desteklenmektedir (Laurichesse ve Privat, 2015). Yazılım boşluk köprüleme (gap-bridging) özelliğine sahip olup, bu özellik sayesinde veri boşlukları giderilebilmektedir (Laurichesse ve Privat, 2015). Yazılıma ait detaylara CNES'in web sitesinden ulaşılabilir (URL-2).

\section{UYGULAMA}

Bu çalışmada RT-PPP yönteminin performansını değerlendirmek amacıyla IGS RT servis istasyonlarından ISTA istasyonu seçilmiştir. İstasyona ait koordinat değerleri 11:00 UT (20.01.2020) - 12:31 UT (21.01.2020) zaman aralığında elde edilmiştir. İlgili günlerde konum doğruluğunu etkileyebilecek jeomanyetik veya solar fırtına kaynaklı iyonosferde düzensiz bir değişim olup olmadığını incelemek amacıyla jeomanyetik firtına (kp) (URL-3), jeomanyetik aktivite (Dst) (URL-4) ve solar aktivite (F10.7) (URL-4) indis değerleri incelenmiştir (Şekil 1, 2). İyonosferde TEC (Total Electron Content) değerlerinde düzensiz bir değişime sebebiyet verecek bir firtınadan bahsedebilmemiz için indis değerlerinin sınır değerlerini aşması gerekir. Bu sınır değerleri kp, Dst ve F10.7 için sırasıyla 5, -50 nT ve 150 sfu' dur. Şekil 1 ve Şekil 2'de görüldüğü gibi, indis değerleri oldukça düşük seviyede olup, herhangi bir jeomanyetik veya solar firtına durumu söz konusu değildir.

ISTA istasyonuna ait RT koordinat değerleri BKG/BNC, RTKLIB/RTKNAVI PPP-WIZARD, yazılımları kullanılarak 1 saniye epok aralıklarında ITRF 2014 referans sisteminde elde edilmiştir. Yazılımlar kullanılırken seçilen parametreler Tablo 1' de verilmiştir. Tabloda verilen parametreler dışındaki tüm seçenekler için ilgili yazılımın varsayılan ayarları kullanılmıştır. Bu üç yazılımdan PPP-WIZARD AR modunda tamsayı belirsizlik çözümünü gerçekleștirebilmektedir. $\mathrm{Bu}$ nedenle yazılımlara ait koordinatlar elde edilirken BKG/BNC, RTKLIB/RTKNAVI, PPP-WIZARD (AR), PPP-WIZARD şeklinde dört seçenek dikkate alınmıştır. Değerlendirme aşamasında sadece GPS gözlemleri kullanılmıştır. Ardından istasyonun ilgili günlerdeki 24 saatlik gözlem dosyaları CSRS-PPP (The Canadian Spatial Reference System-PPP) servisine gönderilerek statik değerlendirme sonucunda koordinat değerleri elde edilmiştir. Elde edilen bu koordinat değerleri referans koordinatlar olarak alınmiștır. Bu referans koordinatlar ile her bir epokta gerçek zamanlı elde edilen koordinatlar kullanılarak X, Y, Z yer merkezli kartezyen sistemden kuzey (n), doğu (e) ve yukarı (u) bileşenleri ile ifade edilen toposentrik koordinat sistemine dönüşüm gerçekleștirilmiștir. Şekil 3-5'de dört yazılım seçeneğinden elde edilen ve referans değerlerinden olan farkları temsil eden toposentrik sistemdeki koordinat bileşenleri verilmektedir. $\mathrm{Bu}$ bileşenler aynı zamanda yazılımlara ait koordinatların doğruluğunu temsil etmektedir.

Tablo 1. Çalışmada kullanılan yazılım paketleri ve kullanılan seçenekler

\begin{tabular}{|c|c|c|c|}
\hline Parametre & BNC v2.12 & \begin{tabular}{|l|} 
RTKLIB \\
v2.4.3
\end{tabular} & $\begin{array}{l}\text { PPP-WIZARD } \\
\text { v1.4.2 }\end{array}$ \\
\hline $\begin{array}{l}\text { Yükseklik } \\
\text { açısı }\end{array}$ & $15^{\circ}$ & $15^{\circ}$ & $15^{\circ}$ \\
\hline \begin{tabular}{|l}
$\begin{array}{l}\text { Uydu } \\
\text { sistemi }\end{array}$ \\
\end{tabular} & GPS & GPS & GPS \\
\hline Epok aralığı & 1 saniye & 1 saniye & 1 saniye \\
\hline Yöntem & RT-PPP & RT-PPP & RT-PPP \\
\hline $\begin{array}{l}\text { Öncül ZHD } \\
\text { model }\end{array}$ & Saastamoinen & Saastamoinen & Saastamoinen \\
\hline $\begin{array}{l}\begin{array}{l}\text { Haritalama } \\
\text { fonk. (MF) }\end{array} \\
\end{array}$ & $\begin{array}{l}1 / \cos (\mathrm{Z}) \\
\mathrm{Z}: \text { Zenit açısı } \\
\end{array}$ & GMF & \begin{tabular}{|l|}
$1 / \sin (\mathrm{e}) ;$ \\
e: Uydu yük. açısı
\end{tabular} \\
\hline $\begin{array}{l}\text { Koordinat } \\
\text { hesabı }\end{array}$ & Var & Var & Var \\
\hline $\begin{array}{l}\text { Yörünge } \\
\text { saat } \\
\text { düzeltme } \\
\text { akıș adı }\end{array}$ & $\begin{array}{l}\text { IGS-03 } \\
\text { Kalman Filt. }\end{array}$ & $\begin{array}{l}\text { IGS-03 } \\
\text { Kalman Filt. }\end{array}$ & $\begin{array}{l}\text { IGS-03 } \\
\text { Kalman Filt. }\end{array}$ \\
\hline $\begin{array}{l}\text { Yörünge } \\
\text { saat } \\
\text { düzeltme } \\
\text { formatı } \\
\end{array}$ & RTCM-SSR & RTCM-SSR & RTCM-SSR \\
\hline $\begin{array}{l}\text { Yayın } \\
\text { efemerisi }\end{array}$ & RTCM3EPH & RTCMЗЕРН & RTCMЗЕРН \\
\hline $\begin{array}{l}\text { Anten faz } \\
\text { merkezi } \\
\text { düzeltmesi } \\
\end{array}$ & igs14.atx & igs14.atx & igs14.atx \\
\hline $\begin{array}{l}\text { Tamsayı faz } \\
\text { başlangıç } \\
\text { belirsizliği } \\
\text { çözümü }\end{array}$ & Yok & Yok & $\begin{array}{l}\text { PPP-AR } \\
\text { modunda var }\end{array}$ \\
\hline \begin{tabular}{|l|} 
Okyanus \\
yüklenmesi
\end{tabular} & Yok & Yok & Yok \\
\hline \begin{tabular}{|l|} 
Atmosfer \\
yüklenmesi \\
\end{tabular} & Yok & Yok & Yok \\
\hline $\begin{array}{l}\text { Katı yer gel- } \\
\text { git etkisi }\end{array}$ & Var & Var & Var \\
\hline \begin{tabular}{|l} 
Faz \\
dönüklüğü \\
düzeltmesi
\end{tabular} & Var & Var & Var \\
\hline
\end{tabular}



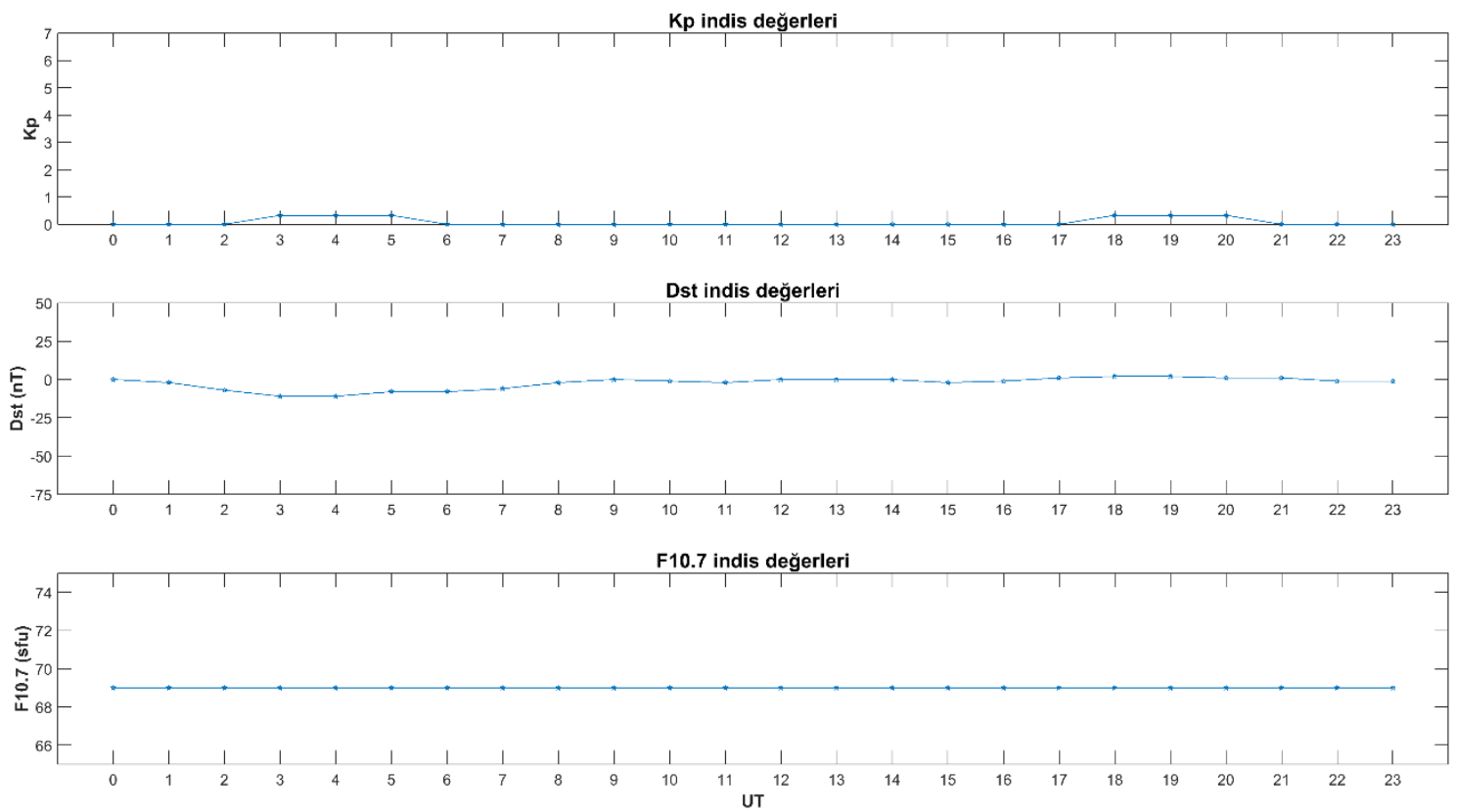

Şekil 1. 20.01.2020 tarihine ait kp, dst, F10.7 değerleri
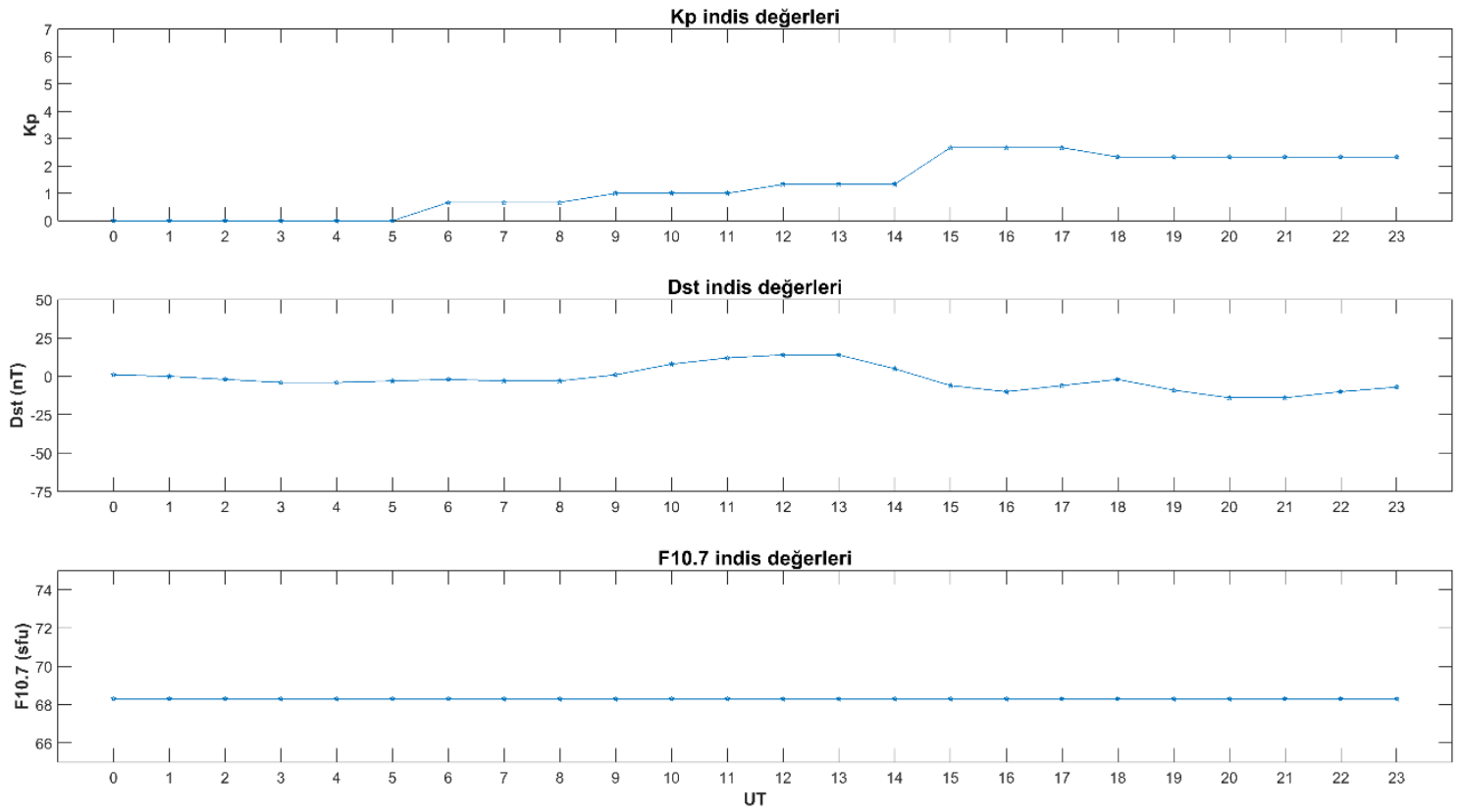

Şekil 2. 21.01.2020 tarihine ait kp, dst, F10.7 değerleri

Şekil 3'te ISTA istasyonunun kuzey (n) bileșenine ait 4 yazılım seçeneğinden elde edilen koordinat değerleri verilmiştir. Yakınsama süresi göz ardı edildiğinde en iyi sonucun RTKLIB yazılımı ile $\pm 3 \mathrm{~cm}$ doğruluğunda elde edildiği görülmüștür. BNC yazılımına ait koordinatlar ise çoğunlukla 8 cm'den küçüktür. PPP-WIZARD (AR) ile ilk yakınsama süresinden sonra 14:17 UT' ye kadar 5 cm'den daha iyi bir doğruluk elde edilmesine rağmen, birçok zaman diliminde koordinat değerleri elde edilememiştir. Ayrıca PPP-WIZARD yazılımı AR seçeneği ile tam sayı belirsizlik çözümünü gerçekleştirdiğinden daha iyi sonuçlar vermesi beklenmesine rağmen, PPP-WIZARD (AR) ve PPPWIZARD sonuçları arasında dikkate değer bir fark olmayıp, bağlantı kesiklikleri ve sonrasındaki yakınsama süreçleri nedeniyle farklar çoğunlukla 30 cm' nin üzerindedir.

Şekil 4'te ise diğer bir yatay bileşen olan doğu (e) bileşenine ait 4 yazılım seçeneğinden elde edilen koordinat değerleri verilmiştir. RTKLIB yazılımı ile oldukça uzun bir yakınsama süresinden sonra $(\sim 15$ saat) $10 \mathrm{~cm}$ 'den daha iyi bir doğruluk elde edilmiştir. 03:00 UT (21.01.2020)' ye kadar olan yakınsama sürecinde koordinat farkları zamanla azalan bir eğilim göstermiştir. $n$ ve $u$ bileșenlerine göre yakınsama süresinin fazla olmasının nedeni gelen düzeltmelerin güncelleme sıklıklarının düşük olmasından kaynaklandığı ve bu durumun e bileşenini daha fazla etkilediği düşünülmektedir. 
BNC yazılımı ile ise yakınsama süreleri hariç doğruluğun $\pm 10 \mathrm{~cm}$ olduğu görülmektedir. $\mathrm{Bu}$ yazılımda bağlantı kesilmesi nedeniyle ikinci bir yakınsama durumu söz konusu olmuştur. Bu yakınsama süreci yaklaşık 1 saati bulmuştur. PPPWIZARD yazılımı ile ise oldukça yüksek koordinat değerleri elde edilmiştir.

ISTA istasyonunun yükseklik bileşenine ilişkin koordinat değerleri incelendiğinde yatay bileşene göre farkların daha büyük olduğu, RTKLIB ve BNC ile 20 cm'lik bir doğruluğun elde edilebildiği görülmüștür (Șekil 5). PPP-WIZARD'a ait iki seçenek kullanılarak elde edilen koordinatlar incelendiğinde farklar zaman zaman $\pm 10 \mathrm{~cm}$ 'den küçük olsa da yatay bileşenlerde olduğu gibi çok sayıda bağlantı kesikliği ve ardından gerçekleșen yakınsama süreleri nedeniyle oldukça büyük seviyelere ulaşmaktadır.

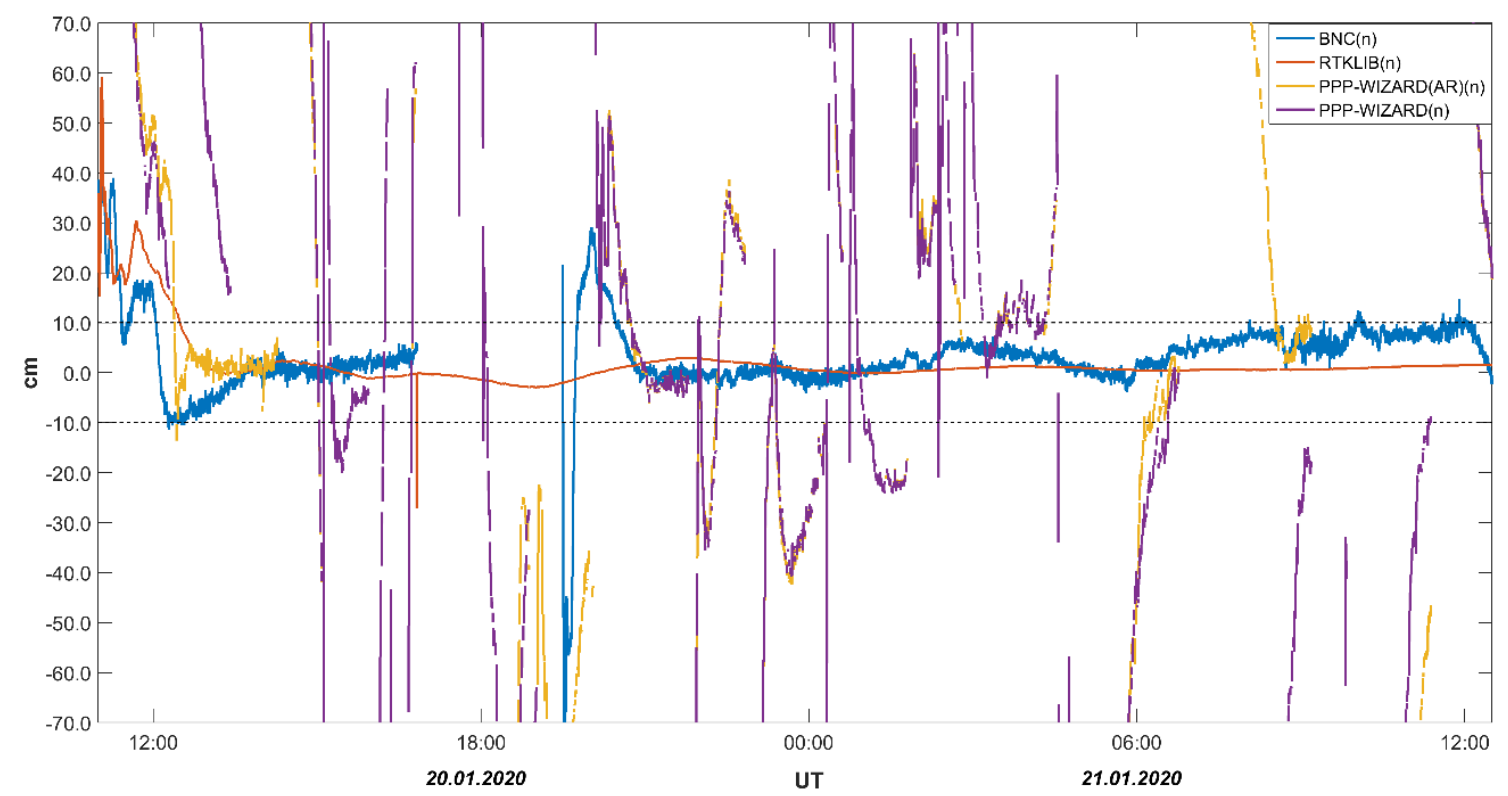

Şekil 3. ISTA istasyonuna ait kuzey bileșeni (n) değerleri

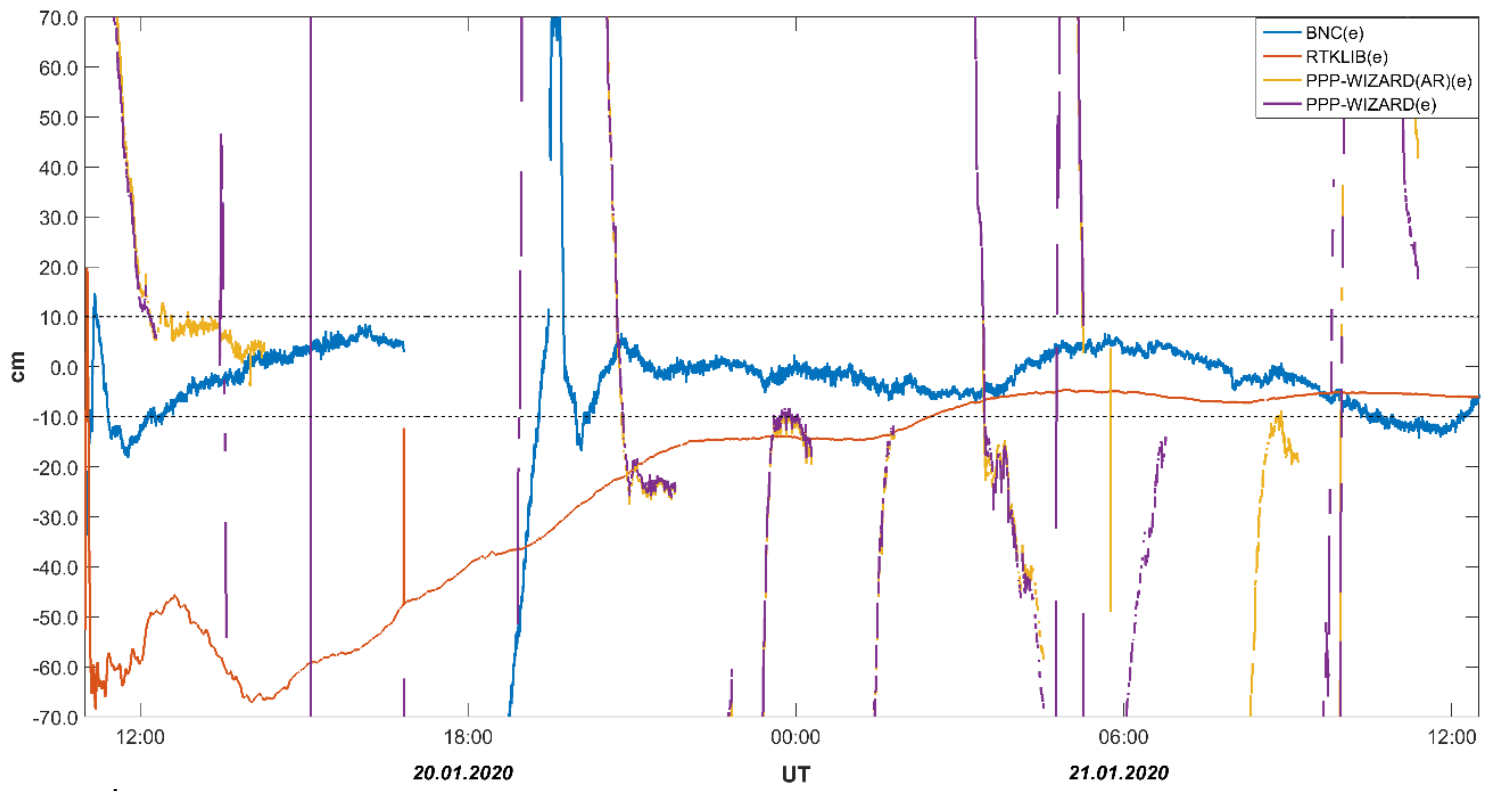

Şekil 4. ISTA İstasyonuna ait doğu bileșeni (e) değerleri 


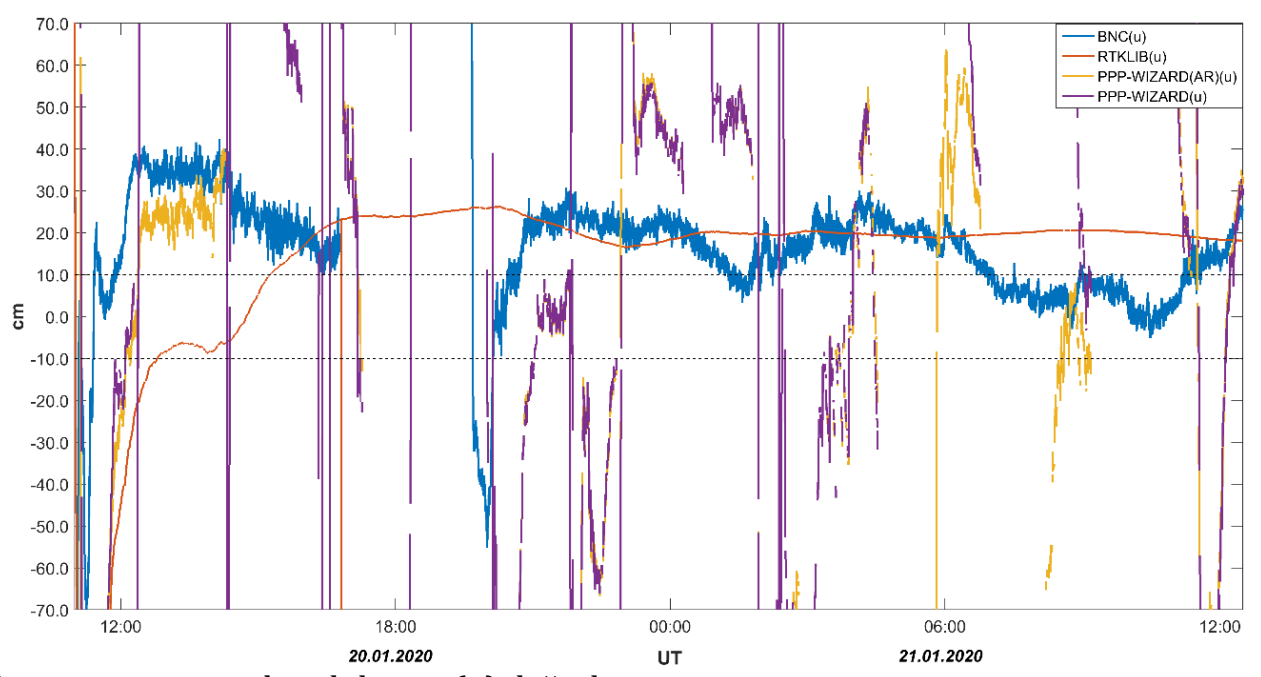

Şekil 5. ISTA İstasyonuna ait yukarı bileşeni (u) değerleri

Sonuçların doğruluk yönünden incelenmesinin yanı sıra, tekrarlılıklarını da test etmek amacıyla tüm koordinat değerleri dikkate alınarak standart sapma (std) değerleri hesaplanmıştır. Std değerlerinin yanı sıra koordinatlara ilişkin temel istatistiki değerlerden mutlak maksimum, mutlak minimum ve ortalama hesaplanmıștır (Tablo 2).

Tablo 2. Toposentrik koordinatlara ilişkin temel istatistiki değerler

\begin{tabular}{|c|c|c|c|c|}
\hline Yazılım & Temel İstatistikler & n (m) & e (m) & u (m) \\
\hline \multirow[t]{4}{*}{ BNC } & Maksimum & 5,351 & 3,555 & 8,460 \\
\hline & Minimum & 0,000 & 0,000 & 0,000 \\
\hline & Ortalama & 0,396 & $-0,208$ & 0,700 \\
\hline & $\overline{\text { Std }}$ & 1,112 & 0,695 & 1,637 \\
\hline \multirow[t]{4}{*}{ RTKLIB } & Maksimum & 0,591 & 0,686 & 3,557 \\
\hline & Minimum & 0,000 & 0,000 & 0,000 \\
\hline & Ortalama & 0,019 & $-0,242$ & 0,121 \\
\hline & $\overline{\text { Std }}$ & 0,056 & 0,210 & 0,257 \\
\hline \multirow{4}{*}{$\begin{array}{l}\text { PPP- } \\
\text { WIZARD } \\
\text { (AR) }\end{array}$} & Maksimum & 13,189 & 9,304 & 27,550 \\
\hline & Minimum & 0,000 & 0,000 & 0,000 \\
\hline & Ortalama & $-0,075$ & $-0,626$ & 1,915 \\
\hline & Std & 1,822 & 2,292 & 6,206 \\
\hline \multirow{4}{*}{$\begin{array}{l}\text { PPP- } \\
\text { WIZARD }\end{array}$} & Maksimum & 13,189 & 9,304 & 30,727 \\
\hline & Minimum & 0,000 & 0,000 & 0,000 \\
\hline & Ortalama & 0,021 & $-0,365$ & 1,787 \\
\hline & $\overline{\text { Std }}$ & 2,303 & 2,656 & 6,450 \\
\hline
\end{tabular}

Tablo 2'de görüldüğü gibi PPP-WIZARD (AR) ve PPP-WIZARD ile gerçek kabul edilen koordinatlarla zaman zaman benzer sonuçlar elde edilebildiği görülse de maksimum, ortalama ve std değerlerinin oldukça yüksek seviyede olduğu görülmektedir. Maksimum değerler incelendiğinde yakınsama süresiyle ilişkili olarak yatay bileșende BNC yazılımı ile 5,351 m (n), 3,555 m (e), RTKLIB yazılımı ile $0,591 \mathrm{~m}(\mathrm{n}), 0,686 \mathrm{~m}(\mathrm{e})$, düşey bileşende ise $\mathrm{BNC}$ ve RTKLIB ile sırasıyla $8,460 \mathrm{~m}$ ve $3,557 \mathrm{~m}$ olduğu görülmektedir. Sonuçların tekrarlılığını test etmek amacıyla std değerleri incelendiğinde en iyi değerlerin tüm bileşenlerde RTKLIB yazılımı kullanılarak elde edildiği görülmektedir.

\section{SONUÇLAR}

Bu çalışmada RT-PPP yönteminin konumlama performansı 3 farklı yazılıma ait 4 seçenek kullanılarak test edilmiştir. ISTA IGS/RTS istasyonunun yaklaşık 25 saatlik gözlem süresine ait 1 saniye epok aralıklı koordinat değerleri doğruluk ve tekrarlılık açısından incelenmiştir. Doğruluk ve tekrarlılık açısından tüm bileşenlerde en iyi sonuçlar RTKLIB yazılımı ile elde edilmiştir. BNC ile elde edilen değerler RTKLIB sonuçlarına yakın olup her iki yazılımla yatay bileșene ait koordinatların düșey bileșene göre doğruluk açısından daha iyi olduğu söylenebilir. PPP-WIZARD yazılımı ile tüm epoklarda koordinat değerleri elde edilememiş olup, doğruluk oldukça düşük seviyededir. Her ne kadar AR seçeneğinin olumlu bir etkisi olacağı beklenmesine rağmen yazılımla ilişkili olarak bu katkı koordinat değerlerinde görülememiştir. Genel olarak elde edilen sonuçlar RT-PPP yöntemin sağladığ konum doğrulu açısından günümüzde yüksek doğruluk gerektiren jeodezik uygulamalarda olmasa da CBS uygulamaları gibi daha düşük doğruluğun yeterli olabileceği haritacılı uygulamalarında kullanılabileceğini göstermiştir. Yöntemin en büyük avantajı referans bir alıcıya gereksinim duyulmadan tek bir GNSS alıcısı ile gerçek zamanlı koordinat elde edilebilmesi olup, günümüzdeki en büyük dezavantajı ise yakınsama süresinin uzun olması ve oluşan bağlantı kesilmeleridir. Yöntemin ve yazilımların performansını kapsamlı olarak test etmek amacıyla farklı zaman dilimlerinde, farklı atmosferik koşullarda, birden fazla istasyon ve uydu sistemi dikkate alınarak değerlendirme işleminin yapılması yerinde olacaktır. $\mathrm{Bu}$ durum farklı istasyonlarda daha iyi bir doğruluğun elde edilip edilemeyeceğini göstermesi açısından önemlidir.

\section{TEŞEKKÜR}

$\mathrm{Bu}$ çalışmada kullanılan BNC yazılımı için Federal Agency for Cartography and Geodesy (BKG)' ye, PPP-WIZARD yazlımı için CNES'e, RTKLIB/RTKNAVI için Takasu Tomoji' ye ve 
değerlendirme aşamasında kullanılan ürünler için IGS-RT servise teşekkür ederiz. Ayrıca makalenin geliștirilmesine yapıcı yorumları ile katkıda bulunan hakemlere teșekkür ederiz.

\section{KAYNAKÇA}

Ahmed, F., Va'clavovic, P., Teferle, F.N., Dous`a, J., Bingley, R., Laurichesse, D. (2016). Comparative analysis of real-time Precise Point Positioning zenith total delay estimates, GPS Solutions, 20, 187-199.

Alçay, S. (2016). Farklı gözlem sürelerinde GPS-PPP ve GPS/GLONASS-PPP yöntemlerinin konum belirleme performanslarının incelenmesi, Afyon Kocatepe Üniversitesi Fen ve Mühendislik Bilimleri Dergisi, 16 (2), 292-302.

Alcay, S., and Turgut, M. (2017). Performance evaluation of real-time precise point positioning method. IOP Conf. Series: Earth and Environmental Science, 95, 032023.

Alçay, S. (2019) Gerçek zamanlı hassas nokta konumlama (RT-PPP) yönteminin konum belirleme performansının doğruluk ve hassasiyet bakımından incelenmesi. Ömer Halisdemir Üniversitesi Mühendislik Bilimleri Dergisi, 8 (1), 121-133.

Alcay, S., Ogutcu, S., Kalayci, I., Yigit, C.O. (2019). Displacement monitoring performance of relative positioning and Precise Point Positioning (PPP) methods using simulation apparatus. Advances in Space Research 63 (5), 1697-1707.

Alkan, R.M., İlçi, V., Ozulu, İ.M., Saka, M.H. (2015). A comparative study for accuracy assessment of PPP technique using GPS and GLONASS in urban areas. Measurement, 69, 1-8.

Choy, S., Zhang, S., Lahaye, F., Héroux, P. (2013). A Comparison between GPS-only and combined GPS+GLONASS Precise Point Positioning. Journal of Spatial Science, 58 (2), 169-190.

Kahveci, M. (2017). Kinematik GNSS ve RTK Cors Ağları, 2. Basım, Nobel Yayıncılık, Ankara.

Krzan, G., and Przestrzelski, P. (2016). GPS/GLONASS Precise Point Positioning with IGS Real-time service product. Acta Geodynamica et Geomaterialia, 13 (1), 69-81.

Laurichesse, D., and Privat, A. (2015). An opensource PPP client implementation for the CNES
PPP-WIZARD demonstrator, Proceedings of the ION GNSS+ 2015, September, Tampa, Florida.

Martin, A., and McGovern, E. (2012). An evaluation of the performance of network RTK GNSS services in Ireland. International Federation of Surveyors (FIG) Working week 6th-10th May 2012.

Ogutcu, S. (2020). Assessing the contribution of Galileo to GPS+GLONASS PPP: Towards full operational capability, Measurement, 151, 107143.

Rizos, C., and Han, S. (2003). Reference station network based RTK systems-concepts and progress, Wuhan University Journal of Natural Sciences. 8, 566-574.

Soycan, M. (2012). A quality evaluation of Precise Point Positioning within the Bernese GPS software version 5.0, Arabian Journal for Science and Engineering, 37, 147-162.

Takasu, T. (2013). RTKLIB ver. 2.4.2 Manual http://www. rtklib. com/prog/manual 2.4.2. pdf.

Tsuji, H., Hatanaka, Y., Hiyama, Y; Yamaguchi, K., Furuya, T., Kawamoto, S., Fukuzaki, Y. (2017). Twenty-year successful operation of GEONET. Bulletin of the Geospatial Information Authority of Japan, 65, 20.

Yigit, C.0, Gikas, V., Alcay, S., Ceylan, A. (2014). Performance evaluation of short to long term GPS, GLONASS and GPS/GLONASS postprocessed PPP, Survey Review, 46 (3), 155-166.

Zhang, K., Wu, F., Wu, S., Rizos, C., Roberts, C., Ge, L., and Ramm, P. (2006). Sparse or dense: Challenges of Australian network RTK. Proceedings of IGNSS 2006.

Zhou, P., Yang, H., Xiao, G., Du, L., Gao, Y. (2019). Estimation of GPS LNAV based on IGS products for real-time PPP. GPS Solutions, 23 (1), 27.

URL-1: https://igs.bkg.bund.de/ntrip/download

URL-2: http://www.ppp-wizard.net/

URL-3:ftp://ftp.gfz-potsdam.de/pub/home/obs /kp-ap/tab/

URL-4:https://omniweb.gsfc.nasa.gov/form/dx1. html 